\title{
Distributions with Exponential Growth and Bochner-Schwartz Theorem for Fourier Hyperfunctions
}

\author{
By
}

\author{
Soon-Yeong CHUNG ${ }^{*}$ and Dohan KIM**
}

\begin{abstract}
Every positive definite Fourier hyperfunction is a Fourier transform of a positive and infra-exponentially tempered measure, which is the generalized Bochner-Schwartz theorem for the Fourier hyperfunctions. To prove this we characterize the distributions with exponential growth via the heat kernel method.

Key words and phrases. Fourier hyperfunction, heat kernel, Bochner-Schwartz theorem, distributions with exponential growth.
\end{abstract}

\section{§0. Introduction}

It is well known in the theory of distributions that

(i ) Every positive distribution is a measure.

(ii) Every positive tempered distribution is a tempered measure.

(iii) (Bochner-Schwartz) Every positive definite (tempered) distribution is the Fourier transform of a positive tempered measure.

Recall that a generalized function $u$ is said to be positive if $u(\varphi) \geq 0$ for every nonnegative test function $\varphi$ and is said to be positive definite (or of positive type in Schwartz [12]) if $u(\varphi * \widetilde{\varphi}) \geq 0$ for any positive test function $\varphi$, where

Communicated by T. Kawai, January $5,1995$.

1991 Mathematics Subject Classification : 46F15, 46F05, 46F10, 35K05

* Department of Mathematics, Duksung Women's University, Seoul 132-714, Korea. Current address: Department of Mathematics, Sogang University, Seoul 121-742, Korea.

** Department of Mathematics, Seoul National University, Seoul 151-742, Korea. Partially supported by KOSEF and GARC. 
$\widetilde{\varphi}(x)=\overline{\varphi(-x)}$. Also, a positive measure $\mu$ is asid ro be tempered if for some $p \geq 0$

$$
\int\left(1+|x|^{2}\right)^{-p} d \mu<\infty
$$

In this paper we will generalize the above theorems to the generalized functions including hyperfunctions, Fourier hyperfunctions, and furthermore Aronszajn traces of analytic solutions of the heat equations, which we will call Aronszajn traces hereafter, as follows.

(i) Every positive hyperfunction is a measure.

(ii) Every positive Fourier hyperfunction is an infra-exponentially tempered measure.

(iii) (Bochner-Schwartz) Every positive definite Fourier hyperfunction is the Fourier transform of a positive and infra-exponentially tempered measure.

(iv) Every positive Aronszajn trace is nothing but a measure.

Here, a positive measure $\mu$ is said to be infra-exponentially tempered if for every $k>0$

$$
\int e^{-k|x|} d \mu<\infty
$$

To prove these main theorems we represent the above generalized functions as the initial values of smooth solutions of heat equations. Using this heat kernel method Matsuzawa gives structure theorems for distributions, hyperfunctions in $[9,11]$ and we give structure theorems for ultradistributions, Fourier hyperfunctions in $[3,10]$ as follows : Let $U(x, t)$ be a solution of the heat equation $(\partial / \partial t-\Delta) U(x, t)=0$ for $t>0$. If $U(x, t)$ is of $O\left(1 / t^{N}\right)$ the initial value $u(x)$ is uniquely determined as a distribution. Also, if $U(x, t)$ is of $O(\exp \epsilon / t)$ $(O(\exp \epsilon(1 / t+|x|)$ respectively) for every $\epsilon>0$ the initial value $u(x)$ is uniquely determined as a hyperfunction (Fourier hyperfunction respectively).

In this paper refining this method more effectively we characterize the distributions with exponential growth and can prove the theorems on the positive hyperfunctions and positive and positive definite Fourier hyperfunctions, which have not been easy due to the sheaf theoretical definition of the hyperfunctions and Fourier hyperfunctions.

In Section 1 we introduce the real version of the space $\mathscr{F}$ of test functions for the Fourier hyperfunctions as in [10] and the space $\mathscr{\bigotimes}_{E}$ of test functions for the infra-exponentially tempered distributions, and their strong dual spaces. Section 2 is devoted to the known results on the representations of distributions 
and Fourier hyperfunctions, and to derive the representation therorem for the space $\mathscr{S}_{E}^{\prime}$ of the infra-exponentially tempered distributions, which are necessary in the next section. In Section 3 as the main section in this paper we prove the main results on the characterizations of the positive hyperfunctions and the positive Fourier hyperfunctions and on the generalization of Bochner-Schwartz theorem for the Fourier hyperfunctions. Finally applying the heat equation method again we prove that every positive Aronszajn trace which can be considered as the initial value of the solutions of the heat equation without any growth condition is also a positive measure in Section 4. We may say that this is the most general theorem on the positive generalized functions.

\section{§1. The Spaces of Generalized Functions}

We use the multi-index notations such as $|\alpha|=\alpha_{1}+\cdots+\alpha_{n}$ for $\alpha \in \mathbf{N}_{0}^{n}$ where $\mathbf{N}_{0}$ is the set of nonnegative integers and $\partial^{\alpha}=\partial_{1}^{\alpha_{1}} \cdots \partial_{n}^{\alpha_{n}}, \partial_{j}=\partial / \partial x_{j}$.

By $C^{\infty}$ we denote the set of all infinitely differentiable functions in $\mathbf{R}^{n}$, by $C_{0}^{\infty}$ the set of $C^{\infty}$ functions with compact support and by \& the Schwartz space of rapidly decreasing functions. Also, we denote by $\mathscr{D}^{\prime}$ the space of distributions in $\mathbf{R}^{n}$ and by $\mathscr{\ell}^{\prime}$ the space of tempered distributions in $\mathbf{R}^{n}$. See [7] for more details on the distributions, Fourier transforms and [8] for hyperfunctions.

We will introduce the real version of the space $\mathscr{F}^{\prime}$ of Fourier hyperfunctions as in [10] and the space $\mathscr{S}_{E}^{\prime}$ of distributions with infra-exponentially tempered growth which are necessary and important throughout this paper.

Definition 1.1 ([10]). (i) We denote by $\mathscr{F}$ the set of all infinitely differentiable functions $\varphi$ in $\mathbf{R}^{n}$ with the property that there exist constants $k, h>0$ such that

$$
\sup _{\substack{x \in \mathbf{R}^{n} \\ \alpha \in \mathbf{N}_{0}^{n}}} \frac{\left|\partial^{\alpha} \varphi(x)\right| \exp k|x|}{h^{|\alpha|} \alpha !}<\infty
$$

(ii) We say that $\varphi_{j} \rightarrow 0$ in $\mathscr{F}$ if there exist $k>0$ and $h>0$ such that

$$
\sup _{\substack{x \in \mathbf{R}^{n} \\ \alpha \in \mathbf{N}_{0}^{n}}} \frac{\left|\partial^{\alpha} \varphi_{j}(x)\right| \exp k|x|}{h^{|\alpha|} \alpha !} \rightarrow 0 \text { as } j \rightarrow \infty
$$

(iii) We denote by $\mathscr{F}^{\prime}$ the strong dual space of $\mathscr{F}$ and call its elements Fourier hyperfunctions. 
Definition 1.2. (i) We denote by $\&_{E}$ the set of all infinitely differentiable functions $\varphi$ in $\mathbf{R}^{n}$ with the property that for any $\alpha \in \mathbb{N}_{0}^{n}$ there exists a positive constant $k$ such that

$$
\sup _{x \in \mathbf{R}^{n}}\left|\partial^{\alpha} \varphi(x)\right| \exp k|x|<\infty .
$$

The function $\varphi \in \mathscr{\bigotimes}_{E}$ is said to be exponentially decreasing on $\mathbf{R}^{n}$.

(ii) We say $\varphi \rightarrow 0$ in $\mathscr{\ell}_{E}$ if for any $N>0$ there exists $k>0$ such that

$$
\sup _{x \in \mathbf{R}^{n}} \sum_{|\alpha| \leq N}\left|\partial^{\alpha} \varphi_{j}(x)\right| \exp k|x| \rightarrow 0 \text { as } j \rightarrow \infty
$$

(iii) We denote by $\mathscr{S}_{E}^{\prime}$ the strong dual space of $\mathscr{S}_{E}$ and call its elements infra-exponentially tempered distributions.

It is easy to see the following topological inclusions:

$$
\begin{gathered}
\mathscr{F} \hookrightarrow \mathscr{S}_{E} \hookrightarrow \mathscr{\&} \\
\mathscr{S}^{\prime} \hookrightarrow \mathscr{S}_{E}^{\prime} \hookrightarrow \mathscr{F}^{\prime} .
\end{gathered}
$$

The space $\mathscr{P}_{*}$ of test functions for Fourier hyperfunctions, which is originally defined by Sato-Kawai, is shown to be isomorphic to the space $\mathscr{F}$ in [10].

Also, we give an equivalent definition for the space $\mathscr{F}$ via Fourier transform as follows.

Theorem $1.3([3])$. The space $\mathscr{F}$ consists of all locally integrable functions $\varphi$ such that

$$
\begin{aligned}
& \sup _{x}|\varphi(x)| \exp k|x|<\infty \\
& \sup _{\xi}|\widehat{\varphi}(\xi)| \exp h|\xi|<\infty
\end{aligned}
$$

for some $h>0$ and $k>0$, where $\widehat{\varphi}(\xi)$ is the Fourier transform of $\varphi$.

Remark. The space $\mathscr{S}_{E}$ is slightly different from $H\left(\mathbb{R}^{n}\right)$ given by Hasumi in [6] and $S_{1}$ given by Gelfand-Shilov in [5]. In fact,

$$
\begin{aligned}
H\left(\mathbf{R}^{n}\right) & =\left\{\varphi \in C^{\infty}\left|{ }^{\forall} \alpha,{ }^{{ }^{*} k}, \sup _{x}\right| \partial^{\alpha} \varphi(x)|\exp k| x \mid<\infty\right\}, \\
S_{1} & =\left\{\varphi \in C^{\infty}\left|{ }^{\exists} k,{ }^{\forall} \alpha, \sup _{x}\right| \partial^{\alpha} \varphi(x)|\exp k| x \mid<\infty\right\}, \\
\mathscr{S}_{E} & =\left\{\left.\varphi \in C^{\infty}\right|^{\forall} \alpha,{ }^{{ }} k, \sup _{x}\left|\partial^{\alpha} \varphi(x)\right| \exp k|x|<\infty\right\} .
\end{aligned}
$$




\section{§2. Representations of $\mathscr{D}^{\prime}, \mathscr{S}^{\prime}{ }_{E}$ and $\mathscr{F}^{\prime}$}

We denote by $E(x, t)$ the $n$-dimensional heat kernel:

$$
\mathrm{E}(x, t)= \begin{cases}(4 \pi t)^{-n / 2} \exp \left(-|x|^{2} / 4 t\right), & t>0 \\ 0, & t \leq 0\end{cases}
$$

Note that $E(\cdot, t)$ belongs to $\mathscr{F}$ for each $t>0$. Thus $U(x, t)=u_{y}(E(x-y, t))$ is well defined for $u \in \mathscr{F}^{\prime}$ or $u \in \mathscr{S}^{\prime}{ }_{E}$.

Consider the following initial value problem:

$$
\left\{\begin{array}{l}
(\partial / \partial t-\Delta) U(x, t)=0,0<t<T, \\
\lim _{t \rightarrow 0^{+}} U(x, t)=u,
\end{array}\right.
$$

where $u$ is a generalized function.

Several authors succeeded in representing various generalized functions such as distributions, hyperfunctions, ultradistributions and Fourier hyperfunctions in $[4,9,10,11]$ as the initial values of solutions of the heat equation.

Theorem 2.1 ([11]). Let $u \in \mathscr{D}^{\prime}$ and $T>0$. Then there exists a $C^{\infty}$ function $U(x, t)$ in $\mathbf{R}^{n} \times(0, T)$ which satisfies the following:

(i ) $(\partial / \partial \mathrm{t}-\Delta) U(x, t)=0$ in $\mathbf{R}^{n} \times(0, T)$.

(ii) For any compact set $K \subset \mathbf{R}^{n}$ there exist $N=N(K)>0$ and $C>0$ such that

$$
\sup _{x \in K}|U(x, t)| \leq C t^{-N}, \quad 0<t<T .
$$

(iii) $\lim _{t \rightarrow 0^{+}} U(x, t)=u$ in $\mathscr{D}^{\prime}$ i.e.,

$$
u(\varphi)=\lim _{t \rightarrow 0^{+}} \int U(x, t) \varphi(x) d x, \varphi \in C_{0}^{\infty}
$$

for every $\varphi \in C_{0}^{\infty}$.

Conversely, if $U(x, t)$ is a $C^{\infty}$ functions in $\mathbf{R}^{n} \times(0, T)$ satisfying (i) and (ii) then there exists unique $u \in \mathscr{D}^{\prime}$ satisfying the relation (iii).

Theorem 2.2([10]). Let $u \in \mathscr{F}^{\prime}$ and $T>0$. Then $U(x, t)=u_{y}(E(x-y, t))$ is a $C^{\infty}$ function in $\mathbf{R}^{n} \times(0, \mathrm{~T})$ and satisfies the following:

(i) $\quad(\partial / \partial t-\Delta) U(x, t)=0$ in $\mathbf{R}^{n} \times(0, \mathrm{~T})$.

(ii) For every $\varepsilon>0$ and every $k>0$ there exists a constant $C>0$ such that 


$$
|U(x, t)| \leq C \exp \left(\frac{\varepsilon}{t}+k|x|\right) \text { in } \mathbf{R}^{n} \times(0, \mathrm{~T}) .
$$

(iii) $\lim _{t \rightarrow 0^{+}} U(x, t)=u$ in $\mathscr{F}^{\prime}$ as in Theorem 2.1 .

Conversely, every $C^{\infty}$ function $U(x, t)$ in $\mathbf{R}^{n} \times(0, \mathrm{~T})$ satisfying (i) and (ii) can be expressed in the form $U(x, t)=u_{y}(E(x-y, t))$ with a unique element $u \in \mathscr{F}^{\prime}$.

In the rest of this section we are going to prove the following representation theorem for the space $\mathscr{X}_{E}^{\prime}$ of infra-exponentially tempered distributions which is essential to prove the main theorems.

Proposition 2.3. For every $\varphi \in \mathscr{S}_{E}$, let

$$
\varphi_{t}(x)=\int_{\mathbf{R}^{n}} E(x-y, t) \varphi(y) d y, \quad t>0
$$

Then $\varphi_{t} \in \oiint_{E}$ for each $t>0$ and $\varphi_{t} \rightarrow \varphi$ in $\oiint_{E}$ as $\mathrm{t} \rightarrow 0^{+}$.

Proof. First, we note that for each $t>0$

$$
\int \mathrm{E}(y, t) d y=1
$$

and for each $\delta>0$ and $k>0$

$$
\int_{|y| \geq \delta} E(y, t) \exp k|y| d y \rightarrow 0
$$

as $t \rightarrow 0^{+}$. It is easy to see that $\varphi_{t} \in \mathscr{S}_{E}$ for each $t>0$. Now we will prove the convergence. Let $\varphi \in \mathscr{S}_{E}$. Then for every $\alpha \in \mathbf{N}_{0}^{n}$ there exist $k>0$ and $C=C(k, \alpha)$ such that

$$
\sup _{x \in \mathbf{R}_{n}}\left|\partial^{\alpha} \varphi(x)\right| \exp k|x| \leq C
$$

and for each $y$ there exists $C^{\prime}>0$ such that

$$
\sup _{x \in \mathbf{R}_{n}}\left|\partial^{\alpha} \varphi(x-y)-\partial^{\alpha} \varphi(x)\right| \exp k|x| \leq C^{\prime}|y|
$$

On the other hand, for each $\delta>0$ we have 


$$
\begin{aligned}
\partial^{\alpha} \varphi(x-y)-\partial^{\alpha} \varphi(x)= & \int_{\mid y_{i} \leq \delta} E(y, t)\left(\partial^{\alpha} \varphi(x-y)-\partial^{\alpha}(x)\right) d y \\
& +\int_{|y| \geq \delta} E(y, t) \partial^{\alpha} \varphi(x-y) d y \\
& -\int_{|y| \geq \delta} E(y, t) \partial^{\alpha} \varphi(x) d y \\
= & I_{1}+I_{2}+I_{3} .
\end{aligned}
$$

Then it follows from (2.4) and (2.7) that

$$
\sup _{x}\left|I_{1}\right| \exp k|x| \leq C^{\prime} \delta
$$

and from (2.5) and (2.6)

$$
\sup _{x}\left|I_{3}\right| \exp k|x| \leq C \int_{|y| \geq \delta} E(y, t) d y \rightarrow 0
$$

as $t \rightarrow 0^{+}$. Also, it follows from (2.5) that

$$
\sup _{x \in \mathbf{R}_{n}}\left|I_{2}\right| \exp k|x| \leq C \int_{|y| \geq \delta} E(y, t) \exp k|y| d y \rightarrow 0
$$

as $t \rightarrow 0^{+}$. Therefore, if we choose $\delta>0$ to be small enough then the estimates (2.8) and (2.9) complete the proof.

Theorem 2.4. Let $u \in \mathscr{S}_{E}^{\prime}$ and $T>0$. Then $U(x, t)=u_{y}(E(x-y, t))$ is a $C^{\infty}$ function in $\mathbf{R}^{n} \times(0, T)$ and satisfies the following:

(i) $(\partial / \partial t-\Delta) U(x, t)=0$ in $\mathbf{R}^{n} \times(0, T)$.

(ii) There exists $N>0$ such that for every $k>0$ and for some $C>0$

$$
|U(x, t)| \leq C t^{-N} \exp k|x| \text { in } \mathbf{R}^{n} \times(0, T) .
$$

(iii) $\lim _{t-0^{+}} U(x, t)=u$ in $\mathscr{S}_{E}^{\prime}$.

Conversely, every $C^{\infty}$ function $U(x, t)$ in $\mathbf{R}^{n} \times(0, T)$ satisfying (i) and (ii) can be expressed in the form $U(x, t)=u_{y}(E(x-y, t))$ with a unique element $u \in \mathscr{S}_{E}^{\prime}$.

Proof. Since $E(x, t)$ belongs to $\oiint_{E}$ for each $t>0, U(x, t)$ is well defined and a $C^{\infty}$ function in $\mathbf{R}^{n} \times(0, T)$ for any $T>0$. Furthermore, $U(x, t)$ satisfies

$$
(\partial / \partial t-\Delta) U(x, t)=0 \text { in } \mathbf{R}^{n} \times(0, T)
$$

On the other hand, $u \in \mathscr{S}_{E}^{\prime}$ implies that there exists $N>0$ such that for every 
$k>0$ and for some $C>0$

$$
|u(\varphi)| \leq C \sup _{x} \sum_{|\alpha| \leq N}\left|\partial^{\alpha} \varphi(x)\right| \exp k|x|, \quad \varphi \in \mathscr{\&}_{E}
$$

Note that that there exists $H>1$ such that

$$
\left|\partial_{x}^{\alpha} E(x, t)\right| \leq H^{|\alpha|+1} t^{-(n+|\alpha|) / 2} \alpha !^{1 / 2} \exp \left(-|x|^{2} / 8 t\right), \quad t>0
$$

Therefore, it follows that

$$
\begin{aligned}
|U(x, t)| & \leq C \sup _{y} \sum_{|\alpha| \leq N}\left|\partial_{x}^{\alpha} E(x-y, t)\right| \exp k|y| \\
& \leq C H^{N+1} \sup _{y} \sum_{|\alpha| \leq N} t^{-(n+|\alpha|) / 2} \alpha !^{1 / 2} \exp \left(-\frac{|x-y|^{2}}{8 t}+k|y|\right) \\
& \leq C^{\prime} t^{-(n+N) / 2} \sup _{y} \exp \left(-\frac{|x-y|^{2}}{8 t}+k|y|\right) \\
& \leq C^{\prime \prime} t^{-(n+N) / 2} \exp k|x|
\end{aligned}
$$

for some constants $C^{\prime}$ and $C^{\prime \prime}$ depending on $k$ and $T$. From this we obtain (ii).

To prove (iii) let $\varphi \in \mathscr{\bigotimes}_{E}$. Then

$$
\int U(x, t) \varphi(x) d x=u_{y}\left(\int E(x-y, t) \varphi(x) d x\right)
$$

by taking limit of the Riemann sum of the first integral. Then it follows from Proposition 2.3 that

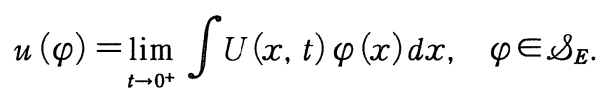

Thus we show that

$$
\lim _{t \rightarrow 0^{+}} U(x, t)=u \text { in }{\varnothing_{E}^{\prime}}
$$

We now prove the converse. For a positive integer $m$ we put

$$
f(t)= \begin{cases}t^{m-1} /(m-1) ! & t \geq 0 \\ 0 & t<0\end{cases}
$$

Multiplying $f$ with a suitable $C^{\infty}$ function with compact support we have the fol- 
lowing relation

$$
(d / d t)^{m} v(t)=\delta(t)+\omega(t)
$$

where $v(t)=f(t)$ for $t \leq T / 4, v(t)=0$ for $t \geq T / 2$ and $\omega(t) \in C^{\infty}(\mathbf{R})$, supp $\omega \subset$ $[T / 4, T / 2]$.

Take $m=N+2$ where $N$ is a constant given in (ii) and consider the following function

$$
\widetilde{U}(x, t)=\int_{0}^{\infty} U(x, t+s) v(s) d s, 0<t<T / 2
$$

Then $\widetilde{U}(x, t)$ is a $C^{\infty}$ function in $\mathbf{R}^{n} \times(0, T / 2)$ and satisfies that for every $k>0$

$$
|\widetilde{U}(x, t)| \leq C \exp k|x| \text { in } \mathbf{R}^{n} \times[0, T / 2) .
$$

This implies that $\widetilde{U}(x, t)$ can be continuously extended to $\mathbf{R}^{n} \times[0, T / 2)$. Moreover, we have

$$
(\partial / \partial t-\Delta) \widetilde{U}(x, t)=0 \text { in } \mathbf{R}^{n} \times(0, T / 2) .
$$

Therefore, it follows from (2.14) and (2.15) that

$$
\begin{aligned}
(-\Delta)^{m} \widetilde{U}(x, t) & =(-d / d t)^{m} \widetilde{U}(x, t) \\
& =U(x, t)+\int_{0}^{\infty} U(x, t+s) \omega(s) d s
\end{aligned}
$$

If we put

$$
H(x, t)=-\int_{0}^{\infty} U(x, t+s) \omega(s) d s
$$

then $H(x, t)$ is also a $C^{\infty}$ solution of heat equation in $\mathbf{R}^{n} \times(0, T / 2)$ which is continuously extended to $\mathbf{R}^{n} \times[0, T / 2)$. Also, for every $k>0$ we have

$$
|H(x, t)| \leq C \exp k|x| \text { in } \mathbf{R}^{n} \times[0, T) .
$$

Furthermore, if we define $g(x)=\widetilde{U}(x, 0)$ and $h(x)=H(x, 0)$ then $g(x)$ and $h(x)$ are continuous on $\mathbf{R}^{n}$ and for every $k>0$

$$
|g(x)| \leq C \exp k|x|, \quad|h(x)| \leq C \exp k|x| .
$$


Because of the uniqueness of the solutions of the heat equation we have

$$
\begin{aligned}
& \widetilde{U}(x, t)=\int E(x-y, t) g(x) d x=g * E \\
& H(x, t)=\int E(x-y, t) h(x) d x=h * E
\end{aligned}
$$

where $*$ denotes the convolution with respect to the $x$ variable. Define $u$ as

$$
u=(-\Delta)^{m} g(x)+h(x) \text {. }
$$

Then, by (2.17) $u$ belongs to $\mathscr{S}_{E}^{\prime}$. The first part of this proof implies that

$$
\lim _{t \rightarrow 0^{+}}(u * E)=u \text { in }{\mathscr{X ^ { \prime }}}_{E}
$$

Hence it remains to show that $U(x, t)=u * E$. In fact, it follows from (2.16) that

$$
\begin{aligned}
u * E & =(-\Delta)^{m}(g * E)+h * E \\
& =(-\Delta)^{m} \widetilde{U}(x, t)+H(x, t) \\
& =U(x, t) .
\end{aligned}
$$

Since the uniqueness of such $u \in{\mathcal{S}^{\prime}}_{E}$ is obvious this completes the proof.

Corollary 2.5. If $u \in{\mathscr{S ^ { \prime }}}_{E}$ then there exist a positive integer $m$ and continuous functions $g(x), h(x)$ on $\mathbf{R}^{n}$ such that for every $k>0$

$$
|g(x)| \leq C \exp k|x|,|h(x)| \leq C \exp k|x|, \quad x \in \mathbb{R}^{n}
$$

and

$$
u=\Delta^{m} g(x)+h(x)
$$

Proof. Let $U(x, t)=u * E$ and $\widetilde{U}(x, t)$ and $H(x, t)$ be as in the above proof. Defining

$$
g(x)=(-1)^{m} \widetilde{U}(x, 0), h(x)=H(x, 0)
$$

we obtain by the similar argument as in the above proof

$$
u=\Delta^{m} g(x)+h(x) .
$$




\section{§3. Positive and Positive Definite Generalized Functions}

In this section we will prove the main theorems on the positive and positive definite generalized functions. First we prove that every positive Fourier hyperfunction and every positive and infra-exponentially tempered distribution is a measure. Also, we will show that every positive hyperfunction is also a measure by an elementary method. Finally, we will show that every positive definite Fourier hyperfunction is the Fourier transform of a positive and infra-exponentially tempered measure, which is the generalized Bochner-Schwartz theorem for the Fourier hyperfunctions.

We first show that every positive element in ${\mathscr{S}^{\prime}}_{E}$ is a measure satisfying some growth condition.

Theorem 3.1. Every positive generalized function in $\mathscr{S}_{E}^{\prime}$ is an infra-exponentially tempered measure.

Conversely, if $\mu$ is a positive and infra-exponentially tempered measure then it defines a positive generalized function $u$ in $\mathscr{S}_{E}^{\prime}$ in a sense that

$$
u(\varphi)=\int \varphi(x) d \mu(x), \quad \varphi \in \mathscr{S}_{E}
$$

Proof. Let $u \in{\mathscr{S ^ { \prime }}}_{E}$ be positive. Then, since ${\mathscr{S}^{\prime}}_{E} \subset \mathscr{D}^{\prime}$ and $u(\varphi) \geq 0$ for every $\varphi$ $\in C_{0}^{\infty}$ with $\varphi \geq 0 u$ must be a measure with

$$
u(\varphi)=\int \varphi d \mu, \quad \varphi \in C_{0}^{\infty}
$$

In order that (3.1) be meaningful for every function $\varphi \in \mathscr{\ell}_{E}$ the measure $\mu$ should be infra-exponentially tempered. To prove this let $\phi(x)$ be a $C^{\infty}$ function with compact support such that $\phi(x)=1$ for $|x| \leq 1$. Consider a sequence $\varphi_{m} \in$ $C_{0}^{\infty}$

$$
\varphi_{m}(x)=\phi\left(\frac{x}{m}\right) \exp \left[-k \sqrt{1+|x|^{2}}\right]
$$

It is clear that $\varphi_{m} \geq 0$ and $\varphi_{m} \rightarrow \exp \left[-k \sqrt{1+|x|^{2}}\right]$ in $\mathscr{\ell}_{E}$ as $m \rightarrow \infty$. Therefore, by the continuity of $u$ on $\mathscr{\ell}_{E}$ there exists $M>0$ such that

$$
\lim _{m \rightarrow \infty} u\left(\varphi_{m}\right) \leq M
$$

So it follows from Fatou's lemma that 


$$
\begin{aligned}
0 \leq \int \exp \left[-k \sqrt{1+|x|^{2}}\right] d \mu & \leq \lim \inf \int \varphi_{m}(x) d \mu \\
& =\lim _{m \rightarrow \infty} u\left(\varphi_{m}\right) \leq M,
\end{aligned}
$$

which implies that $\mu$ is infra-exponentially tempered. This completes the proof since the converse is obvious.

From now on we use the characterizations of generalized functions by the solutions of heat equation developed in Section 2 .

Since the heat kernel $E(x, t) \geq 0$, we have $u * E=u_{y}(E(x-y, t)) \geq 0$ for each $t>0$ if $u$ is a positive element in $\mathscr{S}_{E}^{\prime}$ or $\mathscr{F}^{\prime}$. Conversely, if $U(x, t)$ is a $C^{\infty}$ function satisfying (i) and (ii) in Theorems $2.1,2.2$ and 2.4 and if $U(x, t) \geq 0$ then $U\left(x, 0^{+}\right)$defines a positive element for each case.

We are now in a position to state and prove one of the main theorems which gives the general form of positive Fourier hyperfunctions.

Theorem 3.2. Every positive Fourier hyperfunction is an infra-exponentially tempered measure.

Conversely, if $\mu$ is an infra-exponentially tempered measure, then $\mu$ defines a positive Fourier hyperfunction in a sense that

$$
u(\varphi)=\int \varphi(x) d \mu(x), \quad \varphi \in \mathscr{F}
$$

Proof. By Theorem 3.1 it suffices to show that each positive element in $\mathscr{F}^{\prime}$ belongs to $\mathscr{S}_{E}^{\prime}$. To prove this let $u \in \mathscr{F}^{\prime}$ be positive. Then Theorem 2.2 implies that for every $\varepsilon>0$ and $k>0$ there exists a constant $C>0$ such that

$$
|U(x, t)| \leq C \exp \left(\frac{\varepsilon}{t}+k|x|\right) \text { in } \mathbf{R}^{n} \times(0, T)
$$

where $U(x, t)=u_{y}(E(x-y, t))$. The positivity of $u$ implies that for $0<t<c<T$

$$
\begin{aligned}
0 \leq U(x, t) & =\left(u_{y},(4 \pi t)^{-n / 2} \exp \left(-\frac{|x-y|^{2}}{4 t}\right)\right. \\
& \leq\left(\frac{c}{t}\right)^{n / 2}\left(u_{y},(4 \pi c)^{-n / 2} \exp \left(-\frac{|x-y|^{2}}{4 c}\right)\right) \\
& =\left(\frac{c}{t}\right)^{n / 2}\left(u_{y}, E(x-y, c)\right) \\
& =\left(\frac{c}{t}\right)^{n / 2} U(x, c) .
\end{aligned}
$$


Since $c$ is arbitrary we have

$$
0 \leq U(x, t) \leq C t^{-n / 2} U(x, T)
$$

Hence combining (3.2) and (3.3) we obtain

$$
\begin{aligned}
0 & \leq U(x, t) \leq C t^{-n / 2} \exp \left(\frac{\varepsilon}{T}+k|x|\right) \\
& \leq C(T) t^{-n / 2} \exp k|x| \quad \text { in } \mathbf{R}^{n} \times(0, T) .
\end{aligned}
$$

Then by Theoerm $2.4 u$ belongs to $\mathscr{S}_{E}^{\prime}$. Since the converse is obvious the proof is completed.

We now give a characterization of positive definite Fourier hyperfunctions, which is the generalized Bochner-Schwartz theorem for the Fourier hyperfunctions. Here, we also make use of the heat equation method more effectively which makes the proof possible.

Theorem 3.3. Every positive definite Fourier hyperfunction is the Fourier transform of a positive and infra-exponentially tempered measure $\mu$.

Conversely, the Fourier transform of any positive and infra-exponentially tempered measure defines a positive definite Fourier hyperfunction $u$ in a sense that

$$
u(\varphi)=\int \widehat{\varphi}(\xi) d \mu(\xi), \quad \varphi \in \mathscr{F}
$$

Proof. If $\varphi \in \mathscr{F}$ and $\widetilde{\varphi}(x)=\overline{\varphi(-x)}$ then

$$
\widehat{\varphi * \widetilde{\varphi}}=\widehat{\varphi} \bar{\varphi}
$$

Let $u \in \mathscr{F}^{\prime}$ and $\varphi \in \mathscr{F}$. Since $(\hat{u}, \widehat{\varphi})=(2 \pi)^{n}(u, \varphi)$, the validity of the inequality $(u, \varphi * \widetilde{\varphi}) \geq 0$ for all $\varphi \in \mathscr{F}$ is equivalent to the condition that $(\hat{u}, \phi \bar{\varphi}) \geq$ 0 for all $\phi \in \mathscr{F}$. Here we used the fact that $\mathscr{F}$ is self-dual with respect to the Fourier transformation. Let $V(x, t)=\left(\hat{u}_{y}, E(x-y, t)\right)$.

Since $\hat{u}$ also belongs to $\mathscr{F}^{\prime}$ we have

$$
\begin{aligned}
V(x, t) & =\left(\hat{u}_{y}, E(x-y, t)\right) \\
& =\left(\hat{u}_{y},(4 \pi t)^{-n / 2} \exp \left(-|x-y|^{2} / 4 t\right)\right) \\
& =(4 \pi t)^{-n / 2}\left(\hat{u}_{y}, \exp \left(-|x-y|^{2} / 8 t\right) \overline{\exp \left(-|x-y|^{2} / 8 t\right)}\right. \\
& \geq 0,
\end{aligned}
$$


which shows that $\hat{u}$ is a positive Fourier hyperfunction. Hence Theorem $3.1 \mathrm{im}$ plies that $\hat{u}$ is a positive measure $\mu$ with (3.3), which proves the first part. Since the converse is clear, the proof is completed.

Finally, in the rest of this section we prove that every positive hyperfunction is a measure.

An analytic functional $u$ carried by a compact set $K$ in $\mathbb{R}^{n}$ is defined to be a continuous linear functional on the space of real analytic functions on $K$ with the inductive limit topology. Also, an analytic functional is called positive if $u(\varphi) \geq 0$ for every nonnegative analytic function $\varphi \geq 0$ on $\mathbb{R}^{n}$. A positive hyperfunction on an open set $\Omega$ is considered as a hyperfunction whose restriction on each bounded open subest $V$ of $\Omega$ is represented by a positive analytic functional carried by $\bar{V}$.

Theorem 3.4. Every positive hyperfunction on an open set $\Omega$ is a positive (Radon) measure.

Proof. We have only to show that a positive analytic functional $u$ carried by $\bar{V}$ is a positive measure where $V$ is a bounded open subest of $\Omega$. Let $\varphi$ be a real valued polynomial in $\mathbb{R}^{n}$. Then

$$
\sup _{x \in V}|\varphi(x)| \pm \varphi(x) \geq 0, \quad x \in V
$$

It follows from the positivity of $u$ that

$$
u(1) \sup _{x \in V}|\varphi(x)| \pm u(\varphi) \geq 0
$$

or equivalently that

$$
|u(\varphi)| \leq u(1) \sup _{x \in V}|\varphi(x)|
$$

for every real valued polynomial $\varphi$ in $\mathbb{R}^{n}$. Since every real valued continuous function on $\Omega$ can be uniformly approximated by polynomials on each compact subest of $\Omega u$ can be extended to the space of all continuous functions with topology of compact convergence, i.e., $u$ is a measure. For the general case, if we apply this to $\operatorname{Re} e^{i \theta} \varphi$ where $\theta$ is real and choose $\theta$ so that $e^{i \theta} u(\varphi)$ is real we obtain the same inequality for complex valued $\varphi$. This completes the proof.

In the next section the above theorem will be generalized to the positive 
Aronszajn traces by the heat kernel method.

\section{§4. Positive Temperature Functions}

A $C^{\infty}$ solution of heat equation

$$
(\partial / \partial t-\Delta) u(x, t)=0 \text { in } \mathbf{R}^{n} \times(0, T)
$$

is called a temperature function. In view of Theorem $2.1,2.2$, and 2.3 we expect that each temperature function with some growth condition defines a generalized function. The space of all temperature functions without any growth condition is just the space of Aronszajn traces given in [1].

In this section we will prove that every positive temperature function is a measure which can be the most general theorem on the positive generalized functions. For this the following lemma which is the several variables version of the result in [13] is needed.

Lemma 4.1. Let $U(x, t)$ be a positive temperature function i. e., $C^{\infty}$ function in $\mathbb{R}^{n} \times(0, T)$ such that

(i) $U(x, t) \geq 0$

(ii) $(\partial / \partial t-\Delta) U(x, t)=0$.

Then for any $\delta>0$ and $0<t<T-\delta$ we have

$$
U(x, t+\delta)=\int E(x-y, t) U(y, \delta) d y
$$

We are now in a position to state and prove the main result in this section.

Therorem 4.2. Let $U(x, t)$ be a $C^{\infty}$ function in $\mathbb{R}^{n} \times(0, T)$ such that

(i) $U(x, t) \geq 0$

(ii) $(\partial / \partial t-\Delta) U(x, t)=0$.

Then, for any compact set $K$ of $\mathbf{R}^{n}$ there exist $N>0$ and $C>0$ such that

$$
0 \leq U(x, t) \leq C t^{-N}, \quad x \in K, \quad 0<t<T
$$

In other words, in view of Theorem 2.1 the initial value $U\left(x, 0^{+}\right)$defines a positive distribution, therefore a positive measure. 
Proof. From Lemma 4.1 it follows that for any $\delta>0,0<t<s<T-\delta$,

$$
\begin{aligned}
U(x, t+\delta) & =\int E(x-y, t) U(y, \delta) d y \\
& \leq\left(\frac{s}{t}\right)^{n / 2} \int(4 \pi s)^{-n / 2} \exp \left(-|x-y|^{2} / 4 s\right) U(y, \delta) d y \\
& =\left(\frac{s}{t}\right)^{n / 2} \int E(x-y, s) U(y, \delta) d y \\
& =\left(\frac{s}{t}\right)^{n / 2} U(x, s+\delta)
\end{aligned}
$$

Since $\delta$ is arbitrary the continuity of $U(x, t)$ implies that

$$
\begin{aligned}
0 \leq U(x, t) & \leq\left(\frac{s}{t}\right)^{n / 2} U(x, s), \quad 0<t<s<T \\
& \leq\left(\frac{T}{t}\right)^{n / 2} U(x, T), \quad 0<t<T \\
& \leq C(T) t^{-n / 2}, \quad x \in K, \quad 0<t<T
\end{aligned}
$$

which proves the theorem.

Remark. (i) In fact, Widder [13] proved that every positive temperature function $U(x, t)$ in $\mathbf{R} \times(0, T)$ can be written in the form

$$
U(x, t)=\int_{-\infty}^{\infty} E(x-y, t) d \alpha(y)
$$

where $\alpha$ is an increasing function. But this makes no sense for the sveral variable case, since the Stieltjes measure $d \alpha$ deos not have any meaning. Therefore, the above theorem may be considered as a generalization of the result of Widder.

(ii) In [11] it was shown that every hyperfunction $u$ is a initial value of temperature functions $U(x, t)$ such that for every compact set $K \subset \mathbf{R}^{n}$ and for every $\varepsilon>0$

$$
|U(x, t)| \leq C(K, \epsilon) \exp (\epsilon / t)
$$

for $(x, t) \in K \times(0, T)$. Thus, Theorem 4.2 implies Theorem 3. 5 .

\section{References}

[1] Aronszajn, N., Traces of analytic solutions of the heat equations, Astérisque 2, 3 (1973), 5-68.

[2] Chung, J., Chung, S.-Y. and Kim, D., Une caractérisation de l'espace de Schwartz, C. R. Acad. Sci. Paris Sér. I Math., 316 (1993), 23-25. 
[3] Chung, J., Chung, S.-Y. and Kim, D., A characterization for Fourier hyperfunctions, Publ. RIMS, Kyoto Univ., 30 (1994), 203-208.

[4] Chung, S.-Y., and Kim, D., Representation of quasianalytic ultradistributions, Ark. Mat., 31 (1993), 51-60.

[5] Gelfand, I. M., and Shilov, G. E., Generalized functions II, IV, Academic Press, New York, 1968.

[6] Hasumi, M., Note on the $n$-dimensional tempered ultra-distributions, Tôhoku Math. J., 13 (1961), 94-104.

[7] Hörmander, L., The analysis of linear partial differential operators I, Springer-Verlag, Berlin-New York, 1983.

[8] Kaneko, A., Introduction to hyperfunctions, KTK Sci. Publ., Tokyo, 1988.

[9] Kawai, T., and Matsuzawa, T., On the boundary value of a solution of the heat equation, Publ. RIMS, Kyoto Univ., 25 (1989), 491-498.

[10] Kim, K. H., Chung, S.-Y., and Kim, D., Fourier hyperfunctions as the boundary values of smooth solutions of heat equations, Publ. RIMS, Kyoto Univ., 29 (1993), 289-300.

[11] Matsuzawa, T., A calculus approach to hyperfunctions II, Trans. Amer. Math. Soc., 313 (1990), 619-654.

[12] Schwartz, L.. Théorie des distributions, Hermann, Paris, 1966.

[13] Widder, D. V., The heat equation, Academic Press, New York, 1975. 
